TRANSACTIONS OF THE

AMERICAN MATHEMATICAL SOCIETY

Volume 354, Number 10, Pages 4109-4129

S 0002-9947(02)03000-3

Article electronically published on June 4, 2002

\title{
FUNCTIONAL CALCULUS IN HÖLDER-ZYGMUND SPACES
}

\author{
G. BOURDAUD AND MASSIMO LANZA DE CRISTOFORIS
}

\begin{abstract}
In this paper we characterize those functions $f$ of the real line to itself such that the nonlinear superposition operator $T_{f}$ defined by $T_{f}[g]:=f \circ g$ maps the Hölder-Zygmund space $\mathcal{C}^{s}\left(\mathbf{R}^{n}\right)$ to itself, is continuous, and is $r$ times continuously differentiable. Our characterizations cover all cases in which $s$ is real and $s>0$, and seem to be novel when $s>0$ is an integer.
\end{abstract}

\section{INTRODUCTION AND STATEMENT OF THE MAIN RESULTS}

In this paper we consider the problem of characterizing those functions $f$ defined on the real line, such that the operator $T_{f}: g \mapsto f \circ g$ maps the Hölder-Zygmund space $\mathcal{C}^{s}\left(\mathbf{R}^{n}\right)$ to itself, and is of class $C^{r}$. It is well known that for $0<s<1$, the operator $T_{f}$ maps $\mathcal{C}^{s}\left(\mathbf{R}^{n}\right)$ to itself if and only if $f$ is locally Lipschitz continuous (cf. [4], Drábek [6].) It is also well known that if $s>1$ and if $s$ is not an integer, the operator $T_{f}$ maps $\mathcal{C}^{s}\left(\mathbf{R}^{n}\right)$ to itself if and only if $f$ belongs to $\mathcal{C}^{s}(\mathbf{R})$ locally, and we prove that the same holds also for $s>1, s$ integer. Thus the higher-order Hölder-Zygmund classes behave like the usual Sobolev spaces. Indeed, the acting condition on $W^{m, p}\left(\mathbf{R}^{n}\right)$, for an integer $m>\max (n / p, 1)$, is precisely that $f$ belongs locally to $W^{m, p}(\mathbf{R})$ (cf. [3]). Less clear is the situation in the Zygmund class $\mathcal{C}^{1}$. Indeed, it is not sufficient for $f$ to be locally Lipschitz continuous in order that the operator $T_{f}$ maps $\mathcal{C}^{1}\left(\mathbf{R}^{n}\right)$ to itself. As a matter of fact, there exist continuously differentiable functions for which $T_{f}\left(\mathcal{C}^{1}\left(\mathbf{R}^{n}\right)\right)$ is not contained in $\mathcal{C}^{1}\left(\mathbf{R}^{n}\right)$ !

Theorem 1. Let $s>1$. Then $T_{f}\left(\mathcal{C}^{s}\left(\mathbf{R}^{n}\right)\right) \subseteq \mathcal{C}^{s}\left(\mathbf{R}^{n}\right)$ if and only if $f$ belongs locally to $\mathcal{C}^{s}(\mathbf{R})$.

Theorem 2. $T_{f}\left(\mathcal{C}^{1}\left(\mathbf{R}^{n}\right)\right) \subseteq \mathcal{C}^{1}\left(\mathbf{R}^{n}\right)$ if and only if $f$ is locally Lipschitz continuous and satisfies

$$
f(x+t)+f(x-t)-2 f(x)=O\left(\frac{t}{|\log t|}\right),
$$

as $t \rightarrow 0+$, uniformly on each compact subset of $\mathbf{R}$.

Our plan is the following. First, we recall some basic facts on Hölder-Zygmund spaces. Second, we prove the above theorems. Since Theorem 1 is well known when $s$ is not an integer, we will be concerned mostly with the Zygmund classes $\mathcal{C}^{m}\left(\mathbf{R}^{n}\right)$,

Received by the editors June 13, 2000 and, in revised form, December 21, 2001.

2000 Mathematics Subject Classification. Primary 46E35, $47 \mathrm{H} 30$.

Key words and phrases. Hölder-Zygmund spaces, composition operators.

The authors thank Jean-Pierre Kahane and Winfried Sickel for their help in the preparation of this paper. Massimo Lanza de Cristoforis wishes to thank Gérard Bourdaud and the University of Paris VII for hospitality during the months of September 1998 and of February 2000. 
for $m>0$ an integer, and we shall also prove that our results can be extended in part to the more general Besov spaces $B_{\infty}^{s, q}\left(\mathbf{R}^{n}\right)$. In the third part of the paper, we apply an abstract result of 12 to deduce sufficient conditions for $f$ in order that $T_{f}$ be a map of class $C^{r}$ of $\mathcal{C}^{s}\left(\mathbf{R}^{n}\right)$ into itself, and we prove that such conditions are necessary by exploiting ideas of Drábek [6], of [11], and of Sobolevskij 16], who have considered the case in which $s$ is not an integer.

Notation. We denote by $\mathbf{N}$ the set of all positive integers including 0 , and by $\mathbf{N}^{*}$ the set $\mathbf{N} \backslash\{0\}$. Throughout the paper, $n$ denotes an element of $\mathbf{N}^{*}$. We denote by $\mathbf{R}^{+}$the set of all real numbers $x \geq 0$. We use the letters $M, M^{\prime}, \ldots$ to denote positive constants, depending only on the parameters $n, s$, etc. The exact value of such constants may change from line to line. A constant denoted $A$ will depend also on the various functions $f, g$ under consideration. For any function $f$ on $\mathbf{R}^{n}$ and $a \in \mathbf{R}^{n}$, we define $\tau_{a} f(x):=f(x-a)$. We denote the norm in $L^{p}\left(\mathbf{R}^{n}\right)$ by $\|\cdot\|_{p}$. We denote by $\rho$ a $C^{\infty}$ even function on $\mathbf{R}$ such that $\rho(x)=1$ for $0<x \leq 1 / e$ and $\rho(x)=0$ for $x \geq 1 / 2$. All the functions considered in this paper are real valued.

\section{Preliminaries on FUnCtion SPACES}

\subsection{Sobolev scales.}

Definition 1. Let $\Omega$ be an open set in $\mathbf{R}^{n}$. A Banach distribution space (BDS) in $\Omega$ is a vector subspace $E$ of $\mathcal{D}^{\prime}(\Omega)$ endowed with a complete norm such that the canonical injection $j_{E}: E \rightarrow \mathcal{D}^{\prime}(\Omega)$ is continuous.

The Sobolev scale associated with the BDS $E$ is defined by

$$
W^{r}(E):=\left\{f \in \mathcal{D}^{\prime}(\Omega): f^{(\alpha)} \in E, \quad \forall|\alpha| \leq r\right\},
$$

for any $r \in \mathbf{N}$. It is well known that $W^{r}(E)$ is a BDS for the following norm:

$$
\|f\|_{W^{r}(E)}:=\sum_{|\alpha| \leq r}\left\|f^{(\alpha)}\right\|_{E}
$$

(cf., e.g., [5], Kufner, John and Fučik [10]). By the closed graph theorem, we easily deduce the following.

Lemma 1. Let $E$ be a $B D S$ in $\Omega$. If $\mathcal{D}(\Omega) \subseteq E$, then the canonical injection $\mathcal{D}(\Omega) \rightarrow E$ is continuous. Moreover, $\mathcal{D}(\Omega)$ is continuously imbedded in $W^{r}(E)$, for all $r \in \mathbf{N}$.

2.2. Definition of Hölder-Zygmund spaces. The Besov spaces $B_{\infty}^{s, q}\left(\mathbf{R}^{n}\right)$ can be easily described by means of the first and second order moduli of continuity

$$
\begin{gathered}
\omega(f ; t):=\sup _{|h| \leq t, x \in \mathbf{R}^{n}}|f(x+h)-f(x)|, \\
\eta(f ; t):=\sup _{|h| \leq t, x \in \mathbf{R}^{n}}|f(x+h)+f(x-h)-2 f(x)| .
\end{gathered}
$$

If $0<s<2$ and $1 \leq q \leq \infty$, we say that a real-valued measurable function $f$, defined on $\mathbf{R}^{n}$, belongs to $B_{\infty}^{s, q}\left(\mathbf{R}^{n}\right)$ if, for some (and then for all) $\left.\left.a \in\right] 0,+\infty\right]$, one has

$$
\|f\|_{\infty}+\left(\int_{0}^{a}\left(\frac{\eta(f ; t)}{t^{s}}\right)^{q} \frac{d t}{t}\right)^{1 / q}<+\infty
$$


with the understanding, here and in the rest of the paper, that the case $q=+\infty$ is treated with the usual modifications. For $0<s<1$, one proves classically that a real-valued measurable function $f$ defined on $\mathbf{R}^{n}$ belongs to $B_{\infty}^{s, q}\left(\mathbf{R}^{n}\right)$ if and only if

$$
\|f\|_{\infty}+\left(\int_{0}^{a}\left(\frac{\omega(f ; t)}{t^{s}}\right)^{q} \frac{d t}{t}\right)^{1 / q}<+\infty .
$$

Of course any of the above expressions delivers an equivalent norm in the corresponding Besov space. We denote any such norm by $\|\cdot\|_{B_{\infty}^{s, q}\left(\mathbf{R}^{n}\right)}$. When there can be no ambiguity, we simply write $\|\cdot\|$. Let $m<s \leq m+1$, with $m \in \mathbf{N}^{*}$. Then we set

$$
B_{\infty}^{s, q}\left(\mathbf{R}^{n}\right):=W^{m}\left(B_{\infty}^{s-m, q}\left(\mathbf{R}^{n}\right)\right) .
$$

In particular, $B_{\infty}^{s, \infty}\left(\mathbf{R}^{n}\right)$ is called the Hölder-Zygmund space with exponent $s$ and is denoted by $\mathcal{C}^{s}\left(\mathbf{R}^{n}\right)$. For the main properties of the Besov spaces $B_{p}^{s, q}\left(\mathbf{R}^{n}\right)$, with $s \in \mathbf{R}, p, q \in[1,+\infty]$, and of their homogeneous counterparts $\dot{B}_{p}^{s, q}\left(\mathbf{R}^{n}\right)$, we refer to the books of Peetre [14, of Triebel [19], and of Runst and Sickel [15].

We shall also use the Lipschitz-Sobolev spaces $W_{\infty}^{m}\left(\mathbf{R}^{n}\right):=W^{m}\left(L^{\infty}\left(\mathbf{R}^{n}\right)\right)$. The space of $m$ times continuously differentiable functions on $\mathbf{R}^{n}$ is denoted $C^{m}\left(\mathbf{R}^{n}\right)$. The set

$$
C_{b}^{m}\left(\mathbf{R}^{n}\right):=C^{m}\left(\mathbf{R}^{n}\right) \cap W_{\infty}^{m}\left(\mathbf{R}^{n}\right)
$$

is a closed subspace of $W_{\infty}^{m}\left(\mathbf{R}^{n}\right)$. We say that a function $f$ belongs locally to a functional space $E$ if $f \varphi \in E$ for all $\varphi \in \mathcal{D}\left(\mathbf{R}^{n}\right)$. The set of such $f$ 's is denoted $E_{l o c}$. When there is no ambiguity, we omit the domain $\left(\mathbf{R}\right.$ or $\left.\mathbf{R}^{n}\right)$ in our notation for a function space. We note that $B_{\infty}^{s, q}\left(\mathbf{R}^{n}\right) \subseteq W_{\infty}^{m}\left(\mathbf{R}^{n}\right)$ for $s>m$ and that $W_{\infty}^{1}\left(\mathbf{R}^{n}\right)$ is a proper subspace of $\mathcal{C}^{1}\left(\mathbf{R}^{n}\right)$.

2.3. Basic properties. In 1927, A. Marchaud proved the following relation between the moduli $\omega$ and $\eta$ (see Marchaud [13] or Bennett and Sharpley [2, Thm. 4.4]):

$$
\omega(f ; t) \leq M t \int_{t}^{\infty} \eta(f ; u) \frac{d u}{u^{2}}, \quad \forall t>0 .
$$

We note that $\eta(f ; t) \leq 4\|f\|_{\infty}$, if $f$ is bounded. Thus the Marchaud inequality implies that

$$
\omega(f ; t) \leq M t\left(\int_{t}^{1} \eta(f ; u) \frac{d u}{u^{2}}+4\|f\|_{\infty}\right), \quad \text { for } \quad 0<t \leq 1 .
$$

Proposition 1. Let $1<q \leq+\infty$. Then there exists $M>0$ such that

$$
\left(\int_{0}^{1 / e}\left(\frac{\omega(f ; t)}{t|\log t|}\right)^{q} \frac{d t}{t}\right)^{1 / q} \leq M\|f\|_{B_{\infty}^{1, q}\left(\mathbf{R}^{n}\right)}, \quad \forall f \in B_{\infty}^{1, q}\left(\mathbf{R}^{n}\right) .
$$

Proof. We first note that, for $q=\infty$, (2.3) follows immediately from (2.2). Now we assume that $1<q<\infty$. To shorten our notation, we set $\omega(t):=\omega(f ; t)$, 
$\eta(t):=\eta(f ; t)$. By (2.2) we deduce that

$$
\begin{aligned}
& \left(\int_{0}^{1 / e}\left(\frac{\omega(t)}{t|\log t|}\right)^{q} \frac{d t}{t}\right)^{1 / q} \\
& \quad \leq M\left\{\left(\int_{0}^{1 / e}\left(\frac{1}{|\log t|} \int_{t}^{1} \eta(u) \frac{d u}{u^{2}}\right)^{q} \frac{d t}{t}\right)^{1 / q}+\|f\|_{\infty}\right\}
\end{aligned}
$$

By the changes of variables $t=e^{-v}$ and $u=e^{-w}$, and by the Hardy inequality (cf., e.g., Folland [7, Cor. 6.21]), we have

$$
\begin{gathered}
\left(\int_{0}^{1 / e}\left(\frac{1}{|\log t|} \int_{t}^{1} \eta(u) \frac{d u}{u^{2}}\right)^{q} \frac{d t}{t}\right)^{1 / q}=\left(\int_{1}^{\infty}\left(\frac{1}{v} \int_{0}^{v} \eta\left(e^{-w}\right) e^{w} d w\right)^{q} d v\right)^{1 / q} \\
\leq M\left(\int_{0}^{\infty}\left(\eta\left(e^{-v}\right) e^{v}\right)^{q} d v\right)^{1 / q}=M\left(\int_{0}^{1}\left(\frac{\eta(t)}{t}\right)^{q} \frac{d t}{t}\right)^{1 / q} \leq M\|f\|_{B_{\infty}^{1, q}}
\end{gathered}
$$

Remark. For $q=+\infty$, the inequality 2.3) has been known for a long time (cf., e.g., Zygmund [21 ch. II, par. 3]). Moreover, as we shall see in Lemma 4, inequality (2.3) is optimal if $q=+\infty$. For $q=1$, inequality (2.3) does not hold, but then a more elementary property is available (cf., e.g., Runst and Sickel [15]):

Proposition 2. The Besov space $B_{\infty}^{1,1}\left(\mathbf{R}^{n}\right)$ is imbedded with continuity in $C_{b}^{1}\left(\mathbf{R}^{n}\right)$.

Proposition 3. Let $1 \leq q \leq \infty, s>0$. Then $B_{\infty}^{s, q}\left(\mathbf{R}^{n}\right)$ is a Banach algebra for the usual multiplication of functions.

Proof. This result is well known - almost straightforward if $s$ is not an integer. For $s$ an integer, it is an immediate consequence of Propositions 1 and 2 and of the following identity:

$$
\begin{aligned}
f(x+h) & g(x+h)+f(x-h) g(x-h)-2 f(x) g(x) \\
= & (f(x+h)+f(x-h)-2 f(x)) g(x) \\
& +f(x+h)(g(x+h)+g(x-h)-2 g(x)) \\
& +(f(x-h)-f(x+h))(g(x-h)-g(x)) .
\end{aligned}
$$

We now introduce an inequality for the second-order modulus of the composition of two functions which turns out to be relevant in our analysis. Inequalities of this type have been known for a long time (cf. Warschawski [20, p. 321], Tamrazov [17, [18].) However, for the sake of completeness, we include a proof.

Proposition 4. For all functions $f: \mathbf{R} \rightarrow \mathbf{R}$ and $g: \mathbf{R}^{n} \rightarrow \mathbf{R}$, the following holds:

$$
\eta(f \circ g ; t) \leq \omega(f ; \eta(g ; t))+\eta(f ; \omega(g ; t)), \quad \forall t>0 .
$$


Proof. Inequality (2.5) is an immediate consequence of the following elementary inequality:

$$
\begin{aligned}
\mid f(g(x+h))+ & f(g(x-h)) \\
\leq \frac{1}{2} \mid f(g(x+h))-f(2 g(x) & -g(x-h))\left|+\frac{1}{2}\right| f(g(x-h))-f(2 g(x)-g(x+h)) \mid \\
+ & \frac{1}{2}|f(g(x+h))+f(2 g(x)-g(x+h))-2 f(g(x))| \\
& +\frac{1}{2}|f(g(x-h))+f(2 g(x)-g(x-h))-2 f(g(x))| .
\end{aligned}
$$

2.4. Approximation by entire functions. In order to formulate our results on the continuity and differentiability of $T_{f}$, we need to approximate $f$ by entire functions. We first introduce some notation. Let $\gamma:] 0,1 / e] \rightarrow \mathbf{R}^{+}$be the function defined by

$$
\gamma(t):=\frac{t}{|\log t|}
$$

We denote by $\mathcal{Z}$ the set of functions $f$ of a real variable such that $\eta(f ; t)=O(\gamma(t))$ as $t \rightarrow 0+$. For a function $f \in \mathcal{Z}$, we set

$$
\|f\|_{\mathcal{Z}}:=\sup _{0<t \leq 1 / e} \frac{\eta(f ; t)}{\gamma(t)} .
$$

Clearly $\mathcal{Z} \cap W_{\infty}^{1}$ becomes a Banach space if endowed with the norm

$$
\|f\|_{\infty}+\left\|f^{\prime}\right\|_{\infty}+\|f\|_{\mathcal{Z}}
$$

We denote by $C_{u b}^{m}(\mathbf{R})$ the set of real-valued functions $f$ of class $C^{m}$ on $\mathbf{R}$ such that $f^{(k)}$ is a uniformly continuous bounded function, for $k=0, \ldots, m$. It is easily seen that $C_{u b}^{m}(\mathbf{R})$ is a closed subspace of $W_{\infty}^{m}(\mathbf{R})$. We set

$$
C_{b}^{\infty}(\mathbf{R}):=\bigcap_{m \geq 0} C_{b}^{m}(\mathbf{R})=\bigcap_{m \geq 0} C_{u b}^{m}(\mathbf{R}) .
$$

We note that $C_{b}^{\infty}(\mathbf{R})$ is included in all the function spaces which we have previously defined on $\mathbf{R}$.

Let $\mathcal{H}_{\infty}$ be the set of functions $f \in L^{\infty}(\mathbf{R})$ such that $\widehat{f}$ is a compactly supported distribution. Let $\left(\varphi_{j}\right)_{j \geq 1}$ be an approximation of unity defined by

$$
\varphi_{j}(x):=j \varphi(j x),
$$

where $\widehat{\varphi} \in \mathcal{D}(\mathbf{R})$ and $\widehat{\varphi}(\xi)=1$ in a neighborhood of 0 . If $f \in \mathcal{H}_{\infty}$, then $f$ is the restriction to $\mathbf{R}$ of an entire function and $f=f * \varphi_{j}$ for $j$ sufficiently large. Thus we easily deduce that

$$
\mathcal{H}_{\infty} \subseteq C_{b}^{\infty}(\mathbf{R})
$$

We shall use the following two elementary formulas:

$$
f(x+t)-f(x)=t \int_{0}^{1} f^{\prime}(x+t u) d u
$$


which holds for all functions $f$ in $C^{1}(\mathbf{R})$, and

$$
f(x+t)+f(x-t)-2 f(x)=2 t^{2} \int_{0}^{1} \int_{0}^{1} f^{\prime \prime}(x-t u+2 t u v) u d u d v,
$$

which holds for all functions $f$ in $C^{2}(\mathbf{R})$.

Proposition 5. The following statements hold.

1. Let $m \in \mathbf{N}$. Then $C_{u b}^{m}(\mathbf{R})$ is the closure of $\mathcal{H}_{\infty}$ and of $C_{b}^{\infty}(\mathbf{R})$ in $W_{\infty}^{m}(\mathbf{R})$.

2. Let $m \in \mathbf{N}$. Then the set of $f \in C_{u b}^{m+1}(\mathbf{R})$ such that

$$
\lim _{t \rightarrow 0+}\left(\sup _{x \in \mathbf{R}} \frac{\left|f^{(m)}(x+t)+f^{(m)}(x-t)-2 f^{(m)}(x)\right|}{\gamma(t)}\right)=0
$$

is the closure of $\mathcal{H}_{\infty}$ and of $C_{b}^{\infty}(\mathbf{R})$ in $W^{m}\left(\mathcal{Z} \cap W_{\infty}^{1}\right)$.

3. Let $m \in \mathbf{N}^{*}, m<s<m+1$. Then the set of $f \in C_{u b}^{m}(\mathbf{R})$ such that

$$
\lim _{t \rightarrow 0}\left(\sup _{x \in \mathbf{R}} \frac{\left|f^{(m)}(x+t)-f^{(m)}(x)\right|}{|t|^{s-m}}\right)=0
$$

is the closure of $\mathcal{H}_{\infty}$ and of $C_{b}^{\infty}(\mathbf{R})$ in $\mathcal{C}^{s}(\mathbf{R})$.

4. Let $m \in \mathbf{N}^{*}$. Then the set of $f \in C_{u b}^{m-1}$ such that

$$
\lim _{t \rightarrow 0+}\left(\sup _{x \in \mathbf{R}} \frac{\left|f^{(m-1)}(x+t)+f^{(m-1)}(x-t)-2 f^{(m-1)}(x)\right|}{t}\right)=0
$$

is the closure of $\mathcal{H}_{\infty}$ and of $C_{b}^{\infty}(\mathbf{R})$ in $\mathcal{C}^{m}(\mathbf{R})$.

Proof. We consider the first two function spaces and leave the other two to the reader. Indeed, the proofs are similar.

I. Assume that $f_{j} \in C_{b}^{\infty}(\mathbf{R})$ and that $f_{j} \rightarrow f$ in the function space $E$.

For $E=W_{\infty}^{m}(\mathbf{R})$, we obtain that $f_{j}^{(k)} \rightarrow f^{(k)}$ uniformly on $\mathbf{R}(k=0, \ldots, m)$. Then the uniform continuity of $f^{(k)}$ follows.

Let $E=W^{m}\left(\mathcal{Z} \cap W_{\infty}^{1}\right)$. Since $E \subseteq W_{\infty}^{m+1}(\mathbf{R})$, we have $f \in C_{u b}^{m+1}(\mathbf{R})$. If we apply (2.7) to the function $f_{j}^{(m)}$, we obtain

$$
\frac{1}{\gamma(t)}\left|f_{j}^{(m)}(x+t)+f_{j}^{(m)}(x-t)-2 f_{j}^{(m)}(x)\right| \leq\left\|f_{j}^{(m+2)}\right\|_{\infty} t|\log t| \quad(0<t \leq 1 / e) .
$$

Then

$$
\begin{aligned}
\sup _{x \in \mathbf{R}} & \frac{\left|f^{(m)}(x+t)+f^{(m)}(x-t)-2 f^{(m)}(x)\right|}{\gamma(t)} \\
& \leq\left\|f^{(m)}-f_{j}^{(m)}\right\|_{\mathcal{Z}}+\left\|f_{j}^{(m+2)}\right\|_{\infty} t|\log t| .
\end{aligned}
$$

Hence

$$
\limsup _{t \rightarrow 0+}\left(\sup _{x \in \mathbf{R}} \frac{\left|f^{(m)}(x+t)+f^{(m)}(x-t)-2 f^{(m)}(x)\right|}{\gamma(t)}\right) \leq\left\|f^{(m)}-f_{j}^{(m)}\right\|_{\mathcal{Z}} .
$$

Since the right-hand side is arbitrarily small, we conclude that the limit is 0 .

II. For $f \in E$, we consider the sequence $f_{j}:=f * \varphi_{j}$. Since $E \subseteq L^{\infty}(\mathbf{R})$, it can be readily verified that $f_{j} \in \mathcal{H}_{\infty}$.

If $f \in C_{u b}^{m}(\mathbf{R})$, then one proves classically that $f_{j}^{(k)} \rightarrow f^{(k)}$ uniformly for $k=$ $0, \ldots, m$. Hence $f$ belongs to the closure of $\mathcal{H}_{\infty}$ in $W_{\infty}^{m}(\mathbf{R})$. 
Let $f \in C_{u b}^{m+1}(\mathbf{R})$ be such that (2.8) holds. Then $f_{j} \rightarrow f$ in $W_{\infty}^{m+1}(\mathbf{R})$. Moreover,

$$
\begin{aligned}
\left(f^{(m)}-f_{j}^{(m)}\right)(x+t) & +\left(f^{(m)}-f_{j}^{(m)}\right)(x-t)-2\left(f^{(m)}-f_{j}^{(m)}\right)(x) \\
= & \int_{\mathbf{R}^{n}}\left(f^{(m)}(x+t)-f^{(m)}(x-y+t)+f^{(m)}(x-t)\right. \\
& \left.\quad-f^{(m)}(x-y-t)-2 f^{(m)}(x)+2 f^{(m)}(x-y)\right) \varphi_{j}(y) d y,
\end{aligned}
$$

which implies

$$
\eta\left(f^{(m)}-f_{j}^{(m)} ; t\right) \leq 2\|\varphi\|_{1} \eta\left(f^{(m)} ; t\right) .
$$

On the other hand, $\eta\left(f^{(m)}-f_{j}^{(m)} ; t\right) \leq 4\left\|f^{(m)}-f_{j}^{(m)}\right\|_{\infty}$. Let $\varepsilon>0$. By assumption on $f^{(m)}$, there exists $\delta>0$ such that

$$
0<t<\delta \quad \Rightarrow \quad \frac{\eta\left(f^{(m)} ; t\right)}{\gamma(t)} \leq \frac{\varepsilon}{2\|\varphi\|_{1}} .
$$

Since $f_{j}^{(m)} \rightarrow f^{(m)}$ in $L^{\infty}$, there exists $j_{0}$ such that

$$
j \geq j_{0} \Rightarrow\left\|f^{(m)}-f_{j}^{(m)}\right\|_{\infty} \leq \frac{\varepsilon \gamma(\delta)}{4} .
$$

For such $j$ and for all $t \in] 0,1 / e]$, we obtain

$$
\frac{1}{\gamma(t)} \eta\left(f^{(m)}-f_{j}^{(m)} ; t\right) \leq \varepsilon
$$

that is, $\left\|f^{(m)}-f_{j}^{(m)}\right\|_{\mathcal{Z}} \leq \varepsilon$. By inequality (2.6), we easily see that $W_{\infty}^{2}(\mathbf{R})$ is continuously embedded in $\mathcal{Z}$, and thus in $\mathcal{Z} \cap W_{\infty}^{1}$. Then we conclude that $f_{j} \rightarrow f$ in $W^{m}\left(\mathcal{Z} \cap W_{\infty}^{1}\right)$.

\section{Functional calculus in Besov spaces $B_{\infty}^{s, q}\left(\mathbf{R}^{n}\right)$}

3.1. The case $0<s<1$. For the sake of completeness, we recall the following well-known result (cf., e.g., [4] and (2.1)).

Theorem 3. Let $0<s<1,1 \leq q \leq \infty$. Then $T_{f}\left(B_{\infty}^{s, q}\left(\mathbf{R}^{n}\right)\right) \subseteq B_{\infty}^{s, q}\left(\mathbf{R}^{n}\right)$ if and only if $f$ is locally Lipschitz continuous. Moreover, there exists a continuous increasing function $\psi: \mathbf{R}^{+} \rightarrow \mathbf{R}^{+}$such that

$$
\|f \circ g\|_{B_{\infty}^{s, q}\left(\mathbf{R}^{n}\right)} \leq\|f\|_{W_{\infty}^{1}(\mathbf{R})} \psi\left(\|g\|_{B_{\infty}^{s, q}\left(\mathbf{R}^{n}\right)}\right),
$$

for all $f \in W_{\infty}^{1}(\mathbf{R})$ and $g \in B_{\infty}^{s, q}\left(\mathbf{R}^{n}\right)$.

3.2. The case $s>1$. We are now going to prove a more general version of Theorem 1.

Theorem 4. Let $s>1,1 \leq q \leq \infty$. Then $T_{f}\left(B_{\infty}^{s, q}\left(\mathbf{R}^{n}\right)\right) \subseteq B_{\infty}^{s, q}\left(\mathbf{R}^{n}\right)$ if and only if $f$ belongs locally to $B_{\infty}^{s, q}(\mathbf{R})$. Moreover, there exists a continuous increasing function $\psi: \mathbf{R}^{+} \rightarrow \mathbf{R}^{+}$such that

$$
\|f \circ g\|_{B_{\infty}^{s, q}\left(\mathbf{R}^{n}\right)} \leq\|f\|_{B_{\infty}^{s, q}(\mathbf{R})} \psi\left(\|g\|_{B_{\infty}^{s, q}\left(\mathbf{R}^{n}\right)}\right),
$$

for all $f \in B_{\infty}^{s, q}(\mathbf{R})$ and $g \in B_{\infty}^{s, q}\left(\mathbf{R}^{n}\right)$. 
Proof. The necessity of $f \in B_{\infty, l o c}^{s, q}(\mathbf{R})$ is easily seen by testing $T_{f}$ on a function $g \in \mathcal{D}\left(\mathbf{R}^{n}\right)$ such that $g(x)=x_{1}$ on some ball. To prove the sufficiency, we can assume that $f$ belongs globally to $B_{\infty}^{s, q}(\mathbf{R})$. Indeed, if $\theta \in \mathcal{D}(\mathbf{R})$ satisfies $\theta(x)=1$ on $g\left(\mathbf{R}^{n}\right)$, then $f \circ g=f \theta \circ g$. Then our proof relies on the following intermediate statement.

Lemma 2. Let $1 \leq q \leq \infty, 0<r \leq 1, s>1$. If $f \in B_{\infty}^{r, q}(\mathbf{R})$, then $T_{f}\left(B_{\infty}^{s, q}\left(\mathbf{R}^{n}\right)\right) \subseteq$ $B_{\infty}^{r, q}\left(\mathbf{R}^{n}\right)$. Moreover, there exists a continuous increasing function $\psi: \mathbf{R}^{+} \rightarrow \mathbf{R}^{+}$ such that

$$
\|f \circ g\|_{B_{\infty}^{r, q}\left(\mathbf{R}^{n}\right)} \leq\|f\|_{B_{\infty}^{r, q}(\mathbf{R})} \psi\left(\|g\|_{B_{\infty}^{s, q}\left(\mathbf{R}^{n}\right)}\right),
$$

for all $f \in B_{\infty}^{r, q}(\mathbf{R})$ and $g \in B_{\infty}^{s, q}\left(\mathbf{R}^{n}\right)$.

Proof. It clearly suffices to consider the case $1<s<2$.

1. Let $0<r<1$. The continuous imbedding $B_{\infty}^{s, q}\left(\mathbf{R}^{n}\right) \subseteq W_{\infty}^{1}\left(\mathbf{R}^{n}\right)$ implies that

$$
\omega(g ; t) \leq M\|g\| t
$$

Hence $\omega(f \circ g ; t) \leq \omega(f ; M\|g\| t)$ and

$$
\begin{aligned}
& \left(\int_{0}^{\infty}\left(\frac{\omega(f \circ g ; t)}{t^{r}}\right)^{q} \frac{d t}{t}\right)^{1 / q} \\
& \quad \leq M^{\prime}\|g\|^{r}\left(\int_{0}^{\infty}\left(\frac{\omega(f ; u)}{u^{r}}\right)^{q} \frac{d u}{u}\right)^{1 / q} \leq M^{\prime}\|g\|^{r}\|f\|,
\end{aligned}
$$

which gives us $\|f \circ g\| \leq\|f\|\left(1+M\|g\|^{r}\right)$.

2. Let $r=1$. The imbeddings $B_{\infty}^{s, q}\left(\mathbf{R}^{n}\right) \subseteq \mathcal{C}^{s}\left(\mathbf{R}^{n}\right)$ and $B_{\infty}^{1, q}(\mathbf{R}) \subseteq B_{\infty}^{1 / s, q}(\mathbf{R})$ yield the following inequality:

$$
\begin{aligned}
& \left(\int_{0}^{\infty}\left(\frac{\omega(f ; \eta(g ; t))}{t}\right)^{q} \frac{d t}{t}\right)^{1 / q} \leq\left(\int_{0}^{\infty}\left(\frac{\omega\left(f ; M\|g\| t^{s}\right)}{t}\right)^{q} \frac{d t}{t}\right)^{1 / q} \\
& =s^{-1 / q}(M\|g\|)^{1 / s}\left(\int_{0}^{\infty}\left(\frac{\omega(f ; u)}{u^{1 / s}}\right)^{q} \frac{d u}{u}\right)^{1 / q} \leq M^{\prime}\|g\|^{1 / s}\|f\| .
\end{aligned}
$$

On the other hand, (3.3) implies that

$$
\left(\int_{0}^{\infty}\left(\frac{\eta(f ; \omega(g ; t))}{t}\right)^{q} \frac{d t}{t}\right)^{1 / q} \leq M\|g\|\left(\int_{0}^{\infty}\left(\frac{\eta(f ; u)}{u}\right)^{q} \frac{d u}{u}\right)^{1 / q} \leq M\|g\|\|f\| .
$$

Then, from Proposition 4, we deduce that

$$
\|f \circ g\| \leq\|f\|\left(1+M\left(\|g\|+\|g\|^{1 / s}\right)\right) .
$$

Now we go back to the proof of Theorem 4. Let $m<s \leq m+1, m \in \mathbf{N}^{*}$. We show (3.1) by induction on $m$. It is well known that the norm of $f \circ g$ in $B_{\infty}^{s, q}\left(\mathbf{R}^{n}\right)$ is equivalent to that given by

$$
\|f\|_{\infty}+\sum_{j=1}^{n}\left\|\left(f^{\prime} \circ g\right) \partial_{j} g\right\|_{B_{\infty}^{s-1, q}\left(\mathbf{R}^{n}\right)} .
$$

According to Proposition 3, it suffices to estimate

$$
\left\|f^{\prime} \circ g\right\|_{B_{\infty}^{s-1, q}\left(\mathbf{R}^{n}\right)}\|g\|_{B_{\infty}^{s, q}\left(\mathbf{R}^{n}\right)} .
$$


For $m=1$, we apply Lemma 2 to $f^{\prime} \circ g$. For $m>1$, we apply the inductive assumption to $f^{\prime}$ and $g$.

3.3. The space $B_{\infty}^{1,1}\left(\mathbf{R}^{n}\right)$.

Theorem 5. $T_{f}\left(B_{\infty}^{1,1}\left(\mathbf{R}^{n}\right)\right) \subseteq B_{\infty}^{1,1}\left(\mathbf{R}^{n}\right)$ if and only if $f$ belongs locally to $B_{\infty}^{1,1}(\mathbf{R})$. Moreover, there exists $M>0$ such that

$$
\|f \circ g\|_{B_{\infty}^{1,1}\left(\mathbf{R}^{n}\right)} \leq M\|f\|_{B_{\infty}^{1,1}(\mathbf{R})}\left(\|g\|_{B_{\infty}^{1,1}\left(\mathbf{R}^{n}\right)}+1\right),
$$

for all $f \in B_{\infty}^{1,1}(\mathbf{R})$ and $g \in B_{\infty}^{1,1}\left(\mathbf{R}^{n}\right)$.

Proof. Let $f \in B_{\infty}^{1,1}(\mathbf{R})$ and $g \in B_{\infty}^{1,1}\left(\mathbf{R}^{n}\right)$. From Propositions 2 and 4, we deduce that

$$
\frac{1}{t} \eta(f \circ g, t) \leq\left\|f^{\prime}\right\|_{\infty} \frac{1}{t} \eta(g ; t)+\frac{1}{t} \eta\left(f ; M t\|\nabla g\|_{\infty}\right) .
$$

By taking the norms in $L^{1}(] 0,+\infty\left[, t^{-1} d t\right)$ in the previous inequality, we obtain (3.4).

3.4. The case $s=1, q>1$ : a sufficient condition.

Theorem 6. Let $1<q \leq+\infty$. If $f$ is locally Lipschitz continuous and satisfies condition (1.1) as $t \rightarrow 0+$, uniformly on each compact subset of $\mathbf{R}$, then $T_{f}\left(B_{\infty}^{1, q}\left(\mathbf{R}^{n}\right)\right) \subseteq B_{\infty}^{1, q}\left(\mathbf{R}^{n}\right)$. Moreover, there exists a continuous increasing function $\psi: \mathbf{R}^{+} \rightarrow \mathbf{R}^{+}$such that

$$
\|f \circ g\|_{B_{\infty}^{1, q}\left(\mathbf{R}^{n}\right)} \leq\|f\|_{\mathcal{Z} \cap W_{\infty}^{1}} \psi\left(\|g\|_{B_{\infty}^{1, q}\left(\mathbf{R}^{n}\right)}\right),
$$

for all $f \in \mathcal{Z} \cap W_{\infty}^{1}$ and $g \in B_{\infty}^{1, q}\left(\mathbf{R}^{n}\right)$.

Proof. As in the proof of Theorem 4, we can assume that $f$ belongs to $\mathcal{Z} \cap W_{\infty}^{1}$ (see also (2.4l) ). We choose the following norm in $B_{\infty}^{1, q}\left(\mathbf{R}^{n}\right)$ :

$$
\|g\|:=\|g\|_{\infty}+\left(\int_{0}^{1 / e}\left(\frac{\eta(g ; t)}{t}\right)^{q} \frac{d t}{t}\right)^{1 / q} .
$$

According to Proposition 1, we have $\omega(g ; t)=\kappa(t) t|\log t|$, with

$$
\left(\int_{0}^{1 / e} \kappa(t)^{q} \frac{d t}{t}\right)^{1 / q} \leq M\|g\| .
$$

Since $B_{\infty}^{1, q}\left(\mathbf{R}^{n}\right) \subseteq \mathcal{C}^{1}\left(\mathbf{R}^{n}\right)$, we also have

$$
\sup _{0<t \leq 1 / e} \kappa(t) \leq M\|g\|
$$

By elementary computations, we can show that there exists a continuous decreasing function $\left.\left.w: \mathbf{R}^{+} \rightarrow\right] 0,1 / e\right]$, depending only on $M$, such that

$$
M\|g\| t|\log t| \leq 1 / e \quad \text { and } \quad|\log t| \geq 4 \log (M\|g\|),
$$

for all $t \in] 0, w(\|g\|)]$. Since

$$
\left.\left.1+\frac{\log |\log t|}{\log t} \geq 1 / 2, \quad \forall t \in\right] 0,1 / e\right]
$$

we deduce that

$$
\frac{1}{t} \eta(f ; \omega(g ; t)) \leq \kappa(t)\left|1+\frac{\log |\log t|}{\log t}+\frac{\log \kappa(t)}{\log t}\right|^{-1}\|f\|_{\mathcal{Z}} \leq 4 \kappa(t)\|f\|_{\mathcal{Z}},
$$


for all $t \in] 0, w(\|g\|)]$. On the other hand, we have

$$
\frac{1}{t} \eta(f ; \omega(g ; t)) \leq \frac{4}{t}\left\|f^{\prime}\right\|_{\infty}\|g\|_{\infty}, \quad \forall t>0 .
$$

Assume that $q<\infty$. Then we obtain

$$
\begin{aligned}
\int_{0}^{1 / e}\left(\frac{1}{t} \eta(f ; \omega(g ; t))\right)^{q} \frac{d t}{t} & \\
\leq 4^{q}\|f\|_{\mathcal{Z}}^{q} \int_{0}^{w(\|g\|)} \kappa(t)^{q} \frac{d t}{t} & +4^{q}\left\|f^{\prime}\right\|_{\infty}^{q}\|g\|_{\infty}^{q} \int_{w(\|g\|)}^{\infty} \frac{d t}{t^{q+1}} \\
& \leq 4^{q}\|f\|_{\mathcal{Z} \cap W_{\infty}^{1}}^{q}\|g\|^{q}\left(M^{q}+w(\|g\|)^{-q}\right) .
\end{aligned}
$$

Hence

$$
\left(\int_{0}^{1 / e}\left(\frac{1}{t} \eta(f ; \omega(g ; t))\right)^{q} \frac{d t}{t}\right)^{1 / q} \leq\|f\|_{\mathcal{Z} \cap W_{\infty}^{1}} \psi(\|g\|),
$$

where $\psi: \mathbf{R}^{+} \rightarrow \mathbf{R}^{+}$is some continuous increasing function. The above inequality is easily seen to be true also for $q=+\infty$. Since

$$
\omega(f ; \eta(g ; t)) \leq\left\|f^{\prime}\right\|_{\infty} \eta(g ; t),
$$

then inequality (2.5) yields (3.5).

3.5. The space $\mathcal{C}^{1}$ : a necessary condition. Let $f: \mathbf{R} \rightarrow \mathbf{R}$ be such that

$$
T_{f}\left(\mathcal{C}^{1}\left(\mathbf{R}^{n}\right)\right) \subseteq \mathcal{C}^{1}\left(\mathbf{R}^{n}\right)
$$

By possibly subtracting $f(0)$, we can assume that $f(0)=0$. We already know that $f$ is locally Lipschitz continuous (cf. [4]). To prove condition (1.1), we observe that, in some sense, the nonlinear operator $T_{f}$ is bounded on $\mathcal{C}^{1}$, and we test $T_{f}$ on appropriate functions. We start by introducing the following variant of a classical lemma (cf., e.g., Katznelson [8, ch. VIII, par. 8.3]).

Lemma 3. If $T_{f}\left(\mathcal{C}^{1}\left(\mathbf{R}^{n}\right)\right) \subseteq \mathcal{C}^{1}\left(\mathbf{R}^{n}\right)$, and $f(0)=0$, then the set

$$
\{f \circ g:\|g\| \leq c, \operatorname{supp} g \subseteq K\}
$$

is bounded in $\mathcal{C}^{1}\left(\mathbf{R}^{n}\right)$, for all compact subsets $K$ of $\mathbf{R}^{n}$ and $c>0$.

Proof. Assume for a contradiction that the statement is false. Then there exist $c>0$ and $R>0$ such that

$$
\left\{f \circ g:\|g\| \leq c, \operatorname{supp} g \subseteq[-R, R]^{n}\right\}
$$

is an unbounded set in $\mathcal{C}^{1}\left(\mathbf{R}^{n}\right)$. Let $\theta \in \mathcal{D}\left(\mathbf{R}^{n}\right)$, with support in $[-2 R, 2 R]^{n}$, such that $\theta(x)=1$ on $[-R, R]^{n}$, and let $M_{\theta}$ be the norm of $g \mapsto g \theta$ as a bounded linear operator on $\mathcal{C}^{1}\left(\mathbf{R}^{n}\right)$. By our contradiction assumption, there exists a sequence $\left(q_{j}\right)$ in $\mathcal{C}^{1}\left(\mathbf{R}^{n}\right)$ satisfying the following conditions:

$$
\operatorname{supp} q_{j} \subseteq[-R, R]^{n}, \quad\left\|q_{j}\right\| \leq c, \quad\left\|f \circ q_{j}\right\|>j M_{\theta} .
$$

Now we set

$$
G_{j}:=5 R_{j} e_{1}+[-R, R]^{n}, \quad g_{j}:=\tau_{5 R j e_{1}} q_{j}, \quad \theta_{j}:=\tau_{5 R j e_{1}} \theta,
$$

where $e_{1}:=(1,0, \ldots, 0)$. Since the norm of $\mathcal{C}^{1}$ is translation invariant, we have

$$
\left\|g_{j}\right\| \leq c, \quad\left\|f \circ g_{j}\right\|>j M_{\theta} .
$$


Since supp $g_{j} \subseteq G_{j}$, we can define $g: \mathbf{R}^{n} \rightarrow \mathbf{R}$ by setting $g(x):=g_{j}(x)$ on $G_{j}$ and $g(x):=0$ out of $\bigcup_{j} G_{j}$. Then it is clear that $g \in \mathcal{C}^{1}\left(\mathbf{R}^{n}\right)$. Since $f(0)=0$, we have $f \circ g_{j}=\left(f \circ g_{j}\right) \theta_{j}=(f \circ g) \theta_{j}$. Then we obtain

$$
j M_{\theta}<\left\|(f \circ g) \theta_{j}\right\| \leq M_{\theta_{j}}\|f \circ g\|=M_{\theta}\|f \circ g\|,
$$

a contradiction.

Lemma 4. The function $\sigma(x):=x \rho(x)|\log | x||$ belongs to $\mathcal{C}^{1}(\mathbf{R})$ (see Section 1 for the definition of $\rho$ ).

Proof. We have

$$
-\sigma^{\prime}(x)=\rho(x) \log |x|+\rho(x)+\rho^{\prime}(x) x \log |x|
$$

in the sense of distributions, and the function $x \mapsto \rho(x)+\rho^{\prime}(x) x \log |x|$ is clearly in $\mathcal{D}(\mathbf{R})$. On the other hand, it is well known that the function $x \mapsto \log |x|$ belongs to $B M O(\mathbf{R})$, which is a subspace of the homogeneous Besov space $\dot{B}_{\infty}^{0, \infty}(\mathbf{R})$ (see [5] Prop. VII. 24, p. 154]). Then it can be readily checked that the function $x \mapsto \rho(x) \log |x|$ belongs to $B_{\infty}^{0, \infty}(\mathbf{R})$. Hence $\sigma^{\prime} \in B_{\infty}^{0, \infty}(\mathbf{R})$ and accordingly $\sigma \in B_{\infty}^{1, \infty}(\mathbf{R})$.

Now we go back to the proof of Theorem 2. We first introduce the auxiliary functions

$$
g_{a}(x):=\left(a+\sigma\left(x_{1}\right)\right) \rho(|x|)=a \rho(|x|)+\sigma\left(x_{1}\right) \rho(|x|), \quad \forall x:=\left(x_{1}, \ldots, x_{n}\right) \in \mathbf{R}^{n} .
$$

Let $I$ be a bounded interval. According to Proposition 3 and Lemma 4, the set $\left\{g_{a}: a \in I\right\}$ is bounded in $\mathcal{C}^{1}\left(\mathbf{R}^{n}\right)$. Moreover, the functions $g_{a}$ have support in a fixed compact subset of $\mathbf{R}^{n}$. Then by Lemma 3, there exists a constant $A>0$ such that

$$
\left\|f \circ g_{a}\right\| \leq A, \quad \forall a \in I .
$$

In particular,

$$
\left|f\left(g_{a}(x)\right)+f\left(g_{a}(-x)\right)-2 f\left(g_{a}(0)\right)\right| \leq A|x|, \quad \forall a \in I, \forall x \in \mathbf{R}^{n} .
$$

Let $0<t \leq 1 / e$. Then there exists $h \in] 0,1 / e]$ such that $t=h|\log h|$. If we take $x=h e_{1}$ in (3.6), we obtain

$$
|f(a+t)+f(a-t)-2 f(a)| \leq A h .
$$

However, we also have

$$
\left.\left.h\left|1+\frac{\log |\log h|}{\log h}\right|^{-1} \geq h, \quad \forall h \in\right] 0,1 / e\right] .
$$

Hence

$$
|f(a+t)+f(a-t)-2 f(a)| \leq A \frac{t}{|\log t|} .
$$


3.6. Remarks. Is there a relation between the differentiability of $f$ and the fact that $T_{f}\left(\mathcal{C}^{1}\left(\mathbf{R}^{n}\right)\right) \subseteq \mathcal{C}^{1}\left(\mathbf{R}^{n}\right)$ ? The two following propositions answer this question in part.

The first proposition, namely Proposition 6, is due to Winfried Sickel (personal communication). Below we provide both our proof and his.

Proposition 6. Let $f \in C^{1}(\mathbf{R})$. If

$$
f^{\prime}(x+h)-f^{\prime}(x)=O\left(|\log | h||^{-1}\right),
$$

for $h \rightarrow 0$, uniformly in $x$ on every compact set of $\mathbf{R}$, then $T_{f}\left(\mathcal{C}^{1}\left(\mathbf{R}^{n}\right)\right) \subseteq \mathcal{C}^{1}\left(\mathbf{R}^{n}\right)$.

Proof. Since $f$ is of class $C^{1}$, it is a fortiori locally Lipschitz continuous. Moreover, by the Mean Value Theorem, (3.8) implies (1.1).

We now turn to the proof of Sickel. Without loss of generality, we assume that $f$ is compactly supported. Let $g \in \mathcal{C}^{1}\left(\mathbf{R}^{n}\right)$. By (3.8) and by Proposition 1, we deduce that

$$
\omega\left(f^{\prime} \circ g\right)(t)=O\left(|\log t|^{-1}\right),
$$

as $t \rightarrow 0+$. Then by exploiting the characterization of pointwise multipliers of $B_{\infty}^{0, \infty}$, we obtain $\left(f^{\prime} \circ g\right) \partial_{x_{j}} g \in B_{\infty}^{0, \infty}\left(\mathbf{R}^{n}\right)$ for all $j=1, \ldots, n$ (cf. Koch and Sickel [9] Lem. 20]). Hence, $f \circ g \in B_{\infty}^{1, \infty}\left(\mathbf{R}^{n}\right)$.

Now, we introduce the second proposition.

Proposition 7. The condition $f \in C^{1}$ is neither necessary nor sufficient in order to have $T_{f}\left(\mathcal{C}^{1}\left(\mathbf{R}^{n}\right)\right) \subseteq \mathcal{C}^{1}\left(\mathbf{R}^{n}\right)$.

Proof. For $\alpha \geq 0$, we consider the function

$$
f_{\alpha}(x):=\frac{|x|}{|\log | x||^{\alpha}} \rho(|x|) .
$$

We have $f_{\alpha}(h)+f_{\alpha}(-h)-2 f_{\alpha}(0)=2 f_{\alpha}(h)$. Hence $f_{\alpha}$ does not satisfy the condition (1.1) for $\alpha<1$. Nevertheless $f_{\alpha}$ is continuously differentiable for $\alpha>0$.

We now exhibit a nondifferentiable function $f$ such that $T_{f}\left(\mathcal{C}^{1}\left(\mathbf{R}^{n}\right)\right) \subseteq \mathcal{C}^{1}\left(\mathbf{R}^{n}\right)$. The following example has been indicated to us by Jean-Pierre Kahane. Let $\left\{\varepsilon_{j}\right\}$ be a sequence in $\{-1,+1\}$ such that

$$
u_{k}:=\sum_{j=1}^{k} \frac{\varepsilon_{j}}{j} \quad(k \geq 1)
$$

is a bounded but not convergent sequence. For example, we could take, for $k \in \mathbf{N}$,

$$
\varepsilon_{j}:=1 \quad \text { if } \quad 2^{2 k} \leq j<2^{2 k+1}, \quad \varepsilon_{j}:=-1 \quad \text { if } \quad 2^{2 k+1} \leq j<2^{2 k+2} .
$$

Let $\theta$ be a $C^{\infty}$, positive, even function, decreasing on $[0, \infty[$, with $\operatorname{supp} \theta=[-1,1]$ and $\theta(0)=1$. Let $g$ be the odd primitive of $\theta$. We define the function $f$ by setting

$$
f(x):=\sum_{j=1}^{\infty} \frac{\varepsilon_{j}}{j} 2^{-j} g\left(2^{j} x\right) .
$$

By (2.7), we have

$$
|g(x+h)+g(x-h)-2 g(x)| \leq \min \left(h^{2}\left\|\theta^{\prime}\right\|_{\infty}, 4\|g\|_{\infty}\right), \quad \forall x, h \in \mathbf{R} .
$$


Hence there exists $A>0$ such that

$$
|f(x+h)+f(x-h)-2 f(x)| \leq A\left(h^{2} \sum_{j=1}^{k} \frac{2^{j}}{j}+\sum_{j=k+1}^{\infty} \frac{2^{-j}}{j}\right),
$$

for all $k \in \mathbf{N}^{*}$. By an elementary computation, we have

$$
\sum_{j=1}^{k} \frac{2^{j}}{j} \leq M \frac{2^{k}}{k}, \quad \sum_{j=k+1}^{\infty} \frac{2^{-j}}{j} \leq M \frac{2^{-k}}{k}, \quad \forall k \in \mathbf{N}^{*} .
$$

Then, for each $h \in] 0,1 / 2]$, we take $k$ such that $2^{-k-1}<h \leq 2^{-k}$. The inequalities (3.10) and (3.11) yield

$$
\left.\left.|f(x+h)+f(x-h)-2 f(x)| \leq A \frac{h}{|\log h|}, \quad \text { for all } \quad h \in\right] 0,1 / 2\right], x \in \mathbf{R} .
$$

We have

$$
f^{\prime}(x)=\sum_{j=1}^{\infty} \frac{\varepsilon_{j}}{j} \theta\left(2^{j} x\right)
$$

in the sense of distributions. Since $\theta\left(2^{j} x\right)=0$ for $2^{j} \geq 1 /|x|$, we see that $f^{\prime}$ is a $C^{\infty}$ function on $\mathbf{R} \backslash\{0\}$. Let $x>0$ and $2^{k} \geq 1 / x$. By an Abel transform, we obtain

$$
\left|f^{\prime}(x)\right|=\left|\sum_{j=1}^{k} \varepsilon_{j} j^{-1} \theta\left(2^{j} x\right)\right|=\left|\sum_{j=1}^{k-1} u_{j}\left(\theta\left(2^{j} x\right)-\theta\left(2^{j+1} x\right)\right)\right| \leq \sup _{j \geq 1}\left|u_{j}\right| .
$$

Hence the derivative of $f$ belongs to $L^{\infty}$. In other words, $f$ is Lipschitz continuous. Now we set

$$
\psi(x):=\frac{g(x)}{x} .
$$

We note that $\psi$ can be extended by continuity to $\mathbf{R}$ and satisfies the following asymptotic properties:

$$
\psi(x)=1+O(|x|) \quad \text { as } \quad|x| \rightarrow 0 \quad, \quad \psi(x)=O\left(|x|^{-1}\right) \quad \text { as } \quad|x| \rightarrow \infty .
$$

Moreover, we have

$$
2^{k} f\left(2^{-k}\right)=u_{k}+\sum_{j=1}^{k} \frac{\varepsilon_{j}}{j}\left(\psi\left(2^{j-k}\right)-1\right)+\sum_{j=k+1}^{\infty} \frac{\varepsilon_{j}}{j} \psi\left(2^{j-k}\right) .
$$

From (3.11) and (3.13), we deduce that

$$
2^{k} f\left(2^{-k}\right)-u_{k}=O\left(\frac{1}{k}\right) \quad \text { as } \quad k \rightarrow+\infty .
$$

Thus $\left(2^{k} f\left(2^{-k}\right)\right)_{k \geq 1}$ is not a convergent sequence, which implies that $f$ is not differentiable in 0 .

Finally, it may be of interest to see that the two conditions of Theorem 2 are mutually independent. The function $f_{1 / 2}$ (cf. (3.9) provides an example of a locally Lipschitz continuous function that does not satisfy (1.1). Now let

$$
f(x):=\sum_{j=1}^{\infty} \frac{1}{j} 2^{-j} \sin \left(2^{j} x\right) .
$$


Exactly as in the preceding proof, we see that $f$ satisfies (3.12). We now show that $f$ cannot be locally Lipschitz continuous. If $f$ were locally Lipschitz continuous, then there would exist $C>0$ such that $|f(x)| \leq C x$, for $0<x \leq 1$. By taking $x=2^{-N}$, with $N \in \mathbf{N}^{*}$, we obtain

$$
\left|\sum_{j=1}^{N} \frac{1}{j} 2^{-j} \sin \left(2^{j-N}\right)\right| \leq C 2^{-N}+\sum_{j>N} \frac{2^{-j}}{j} \leq(C+1) 2^{-N} .
$$

On the other hand, we have $\sin \left(2^{j-N}\right) \geq \frac{2}{\pi} 2^{j-N}$ for $1 \leq j \leq N$. Hence,

$$
\left|\sum_{j=1}^{N} \frac{1}{j} 2^{-j} \sin \left(2^{j-N}\right)\right| \geq \frac{2}{\pi} 2^{-N}\left(\sum_{j=1}^{N} \frac{1}{j}\right)
$$

a contradiction.

\section{Continuity And Differentiability of the Superposition operator}

The following regularity theorem provides a full characterization of the functions $f$ for which $T_{f}$ is a $C^{r}$ mapping on the Hölder-Zygmund space.

Theorem 7. Let $s>0$ and $r \in \mathbf{N}$. Let $f$ be a function from $\mathbf{R}$ to itself. Then the following list provides, for the various values of $s$, a necessary and sufficient condition for the operator $T_{f}$ to be of class $C^{r}$ from $\mathcal{C}^{s}\left(\mathbf{R}^{n}\right)$ to itself.

1. $f$ is of class $C^{r+1}$, if $0<s<1$.

2. $f$ is of class $C^{r+1}$ and

$$
f^{(r)}(x+t)+f^{(r)}(x-t)-2 f^{(r)}(x)=o(\gamma(t)),
$$

as $t \rightarrow 0+$, uniformly on each compact subset of $\mathbf{R}$, if $s=1$.

3. $f$ is of class $C^{r+m}$ and

$$
f^{(r+m)}(x+t)-f^{(r+m)}(x)=o\left(|t|^{s-m}\right)
$$

as $t \rightarrow 0$, uniformly on each compact subset of $\mathbf{R}$, if $m<s<m+1$ for some $m \in \mathbf{N}^{*}$.

4. $f$ is of class $C^{r+s-1}$ and

$$
f^{(r+s-1)}(x+t)+f^{(r+s-1)}(x-t)-2 f^{(r+s-1)}(x)=o(t),
$$

as $t \rightarrow 0+$, uniformly on each compact subset of $\mathbf{R}$, if $s$ is integer and $s>1$.

We note that statements 1 and 3 are variants of known results. For references to the various contributions, we refer to [11] and to the extensive monograph of Appell and Zabreiko [1]. However, we believe that statements 2 and 4 are novel.

As a first step in proving Theorem 7 we introduce an abstract differentiability result for the composition operator of [12], which we apply to the Hölder-Zygmund classes in the next section.

\subsection{An abstract regularity theorem.}

Proposition 8. Let $E$ be a $B D S$ in $\mathbf{R}$. Let $I$ be a bounded open interval of $\mathbf{R}$. Let $\mathcal{R}_{I}$ be the restriction operator from $\mathcal{D}^{\prime}(\mathbf{R})$ to $\mathcal{D}^{\prime}(I)$. Then $E(I):=\mathcal{R}_{I}(E)$, equipped with the quotient norm associated with the map $\mathcal{R}_{I}: E \rightarrow E(I)$, is a BDS in $I$. 
Proof. If $f_{k} \rightarrow f$ in $E$, with $\mathcal{R}_{I}\left(f_{k}\right)=0$, then $f_{k} \rightarrow f$ in $\mathcal{D}^{\prime}(\mathbf{R})$, and accordingly $\mathcal{R}_{I}(f)=0$. Thus the kernel of $\mathcal{R}_{I}: E \rightarrow E(I)$ is a closed subspace of $E$, and accordingly $E(I)$, with the quotient norm, is a Banach space. We have $j_{E(I)} \circ \mathcal{R}_{I}=$ $\mathcal{R}_{I} \circ j_{E}$, and $\mathcal{R}_{I} \circ j_{E}$ is a continuous mapping from $E$ to $\mathcal{D}^{\prime}(I)$. Thus, by definition of the quotient topology, $j_{E(I)}$ continuously maps $E(I)$ to $\mathcal{D}^{\prime}(I)$.

Lemma 5. Let $E$ be a $B D S$ in $\mathbf{R}$ such that $\mathcal{D}(\mathbf{R}) \subseteq E$. Let $\mathcal{P}(\mathbf{R})$ be the set of polynomials in one real variable. If $I$ is a bounded nonempty open interval of $\mathbf{R}$, then $\mathcal{R}_{I}$ is an injection of $\mathcal{P}(\mathbf{R})$ into $E(I)$.

Proof. Let $\theta \in \mathcal{D}(\mathbf{R})$ be such that $\theta(x)=1$ in a neighborhood of $I$. Then, for all $p \in \mathcal{P}(\mathbf{R})$, we have $\theta p \in \mathcal{D}(\mathbf{R}) \subseteq E$, and thus $\mathcal{R}_{I}(p)=\mathcal{R}_{I}(\theta p) \in E(I)$.

Lemma 6. Let $r \in \mathbf{N}$. Let I be a bounded nonempty open interval of $\mathbf{R}$. Let $E$ be a BDS in $\mathbf{R}$ such that $\mathcal{D}(\mathbf{R}) \subseteq E$. Let $P_{r, E}(I)$ denote the closure of $\mathcal{R}_{I}(\mathcal{P}(\mathbf{R}))$ in $W^{r}(E(I))$. Then the following function is a norm on $\mathcal{P}(\mathbf{R})$ :

$$
\|p\|_{r, E, I}:=\left\|\mathcal{R}_{I}(p)\right\|_{W^{r}(E(I))} \quad \forall p \in \mathcal{P}(\mathbf{R}),
$$

and $P_{r, E}(I)$ is a completion of $\left(\mathcal{P}(\mathbf{R}),\|\cdot\|_{r, E, I}\right)$. Moreover, $P_{r_{1}, E}(I)$ is imbedded with continuity in $P_{r_{2}, E}(I)$ if $r_{1}>r_{2}$, and $P_{0, E}(I)$ is imbedded with continuity in $E(I)$.

Proof. The fact that $\|\cdot\|_{r, E, I}$ is a norm on $\mathcal{P}(\mathbf{R})$ is a consequence of Lemma 5 . The other statements are straightforward.

In the following, we denote by $\mathcal{H}$ the set of functions from $\mathbf{R}$ to itself that are restrictions to $\mathbf{R}$ of entire analytic functions, and we denote by $\mathrm{cl}_{E} A$ the closure of a set $A$ in a certain space $E$.

Proposition 9. Let $r \in \mathbf{N}$. Let I be a bounded nonempty open interval of $\mathbf{R}$. Let $E$ be a $B D S$ in $\mathbf{R}$ such that $\mathcal{D}(\mathbf{R}) \subseteq E$. Then

(i) $\mathcal{R}_{I}$ maps continuously $W^{r}(E)$ into $W^{r}(E(I))$, and

(ii) $\mathcal{R}_{I}\left(\operatorname{cl}_{W^{r}(E)}\left(W^{r}(E) \cap \mathcal{H}\right)\right) \subseteq P_{r, E}(I)$.

Proof. From the identity $\left(\mathcal{R}_{I} f\right)^{(j)}=\mathcal{R}_{I}\left(f^{(j)}\right)$ (which holds for all $f \in \mathcal{D}^{\prime}(\mathbf{R})$ ), we deduce that

$$
\left\|\mathcal{R}_{I} f\right\|_{W^{r}(E(I))}=\sum_{j=0}^{r}\left\|\mathcal{R}_{I}\left(f^{(j)}\right)\right\|_{E(I)} \leq \sum_{j=0}^{r}\left\|f^{(j)}\right\|_{E}=\|f\|_{W^{r}(E)} .
$$

We now prove that

$$
\mathcal{R}_{I}(\mathcal{H}) \subseteq P_{r, E}(I)
$$

Then (ii) follows by (i). For $f \in \mathcal{H}$, we consider the Taylor polynomials

$$
p_{l}(x):=\sum_{j=0}^{l} f^{(j)}(0) \frac{x^{j}}{j !} .
$$

Let $\theta \in \mathcal{D}(\mathbf{R})$ be such that $\theta(x)=1$ in a neighborhood of $I$. Since $p_{l} \rightarrow f$, with all its derivatives, uniformly on each compact subset of $\mathbf{R}$, we have $p_{l} \theta \rightarrow f \theta$ in $\mathcal{D}(\mathbf{R})$. Accordingly, by assumption $\mathcal{D}(\mathbf{R}) \subseteq E$ and by Lemma 1 , we have $p_{l} \theta \rightarrow f \theta$ in $W^{r}(E)$. Hence, by (i), we have

$$
\mathcal{R}_{I}\left(p_{l}\right) \rightarrow \mathcal{R}_{I}(f)
$$

in $W^{r}(E(I))$, and accordingly $\mathcal{R}_{I}(f) \in P_{r, E}(I)$. 
By [12, we have the following general result.

Theorem 8. Let all the assumptions of Proposition 9 hold. Let $\mathcal{X}$ be a real commutative Banach algebra with unity. Let $\mathcal{A}$ be a subset of $\mathcal{X}$. Assume there exists an increasing function $\psi$ of $\mathbf{R}^{+}$to itself such that

$$
\|p(x)\|_{\mathcal{X}} \leq\left\|\mathcal{R}_{I} p\right\|_{E(I)} \psi\left(\|x\|_{\mathcal{X}}\right),
$$

for all $(p, x) \in \mathcal{P}(\mathbf{R}) \times \mathcal{A}$. Then there exists a unique map $\Phi$ of $P_{0, E}(I) \times \mathcal{A}$ to $\mathcal{X}$ such that the following two conditions hold:

(i) $\Phi(p, x)=p(x)$, for all $(p, x) \in \mathcal{P}(\mathbf{R}) \times \mathcal{A}$.

(ii) The map $\Phi(\cdot, x)$ is continuous from $P_{0, E}(I)$ to $\mathcal{X}$ for all fixed $x \in \mathcal{A}$.

Furthermore, the map $\Phi(\cdot, x)$ of (ii) is linear and $\Phi$ is continuous from $P_{0, E}(I) \times \mathcal{A}$ to $\mathcal{X}$, and if $x \in \mathcal{A}$ and $f \in P_{0, E}(I)$, with $f=\lim _{j \rightarrow \infty} \mathcal{R}_{I} p_{j}$ in $P_{0, E}(I), p_{j} \in \mathcal{P}(\mathbf{R})$, then

$$
\Phi(f, x)=\lim _{j \rightarrow \infty} p_{j}(x), \quad \text { in } \mathcal{X}
$$

and

$$
\|\Phi(f, x)\|_{\mathcal{X}} \leq\|f\|_{E(I)} \psi\left(\|x\|_{\mathcal{X}}\right) .
$$

If we further assume that $\mathcal{A}$ is open and that $r \geq 1$, then $\Phi$ is of class $C^{r}$ from $P_{r, E}(I) \times \mathcal{A}$ to $\mathcal{X}$.

4.2. Proof of the regularity Theorem 17, Sufficiency. In all this section, we consider some bounded nonempty open interval $I$ and a function $\theta \in \mathcal{D}(\mathbf{R})$ such that $\theta(x)=1$ in a neighborhood of $\operatorname{cl}(I)$. The set

$$
\mathcal{A}:=\left\{g \in \mathcal{C}^{s}\left(\mathbf{R}^{n}\right): g\left(\mathbf{R}^{n}\right) \subseteq I\right\}
$$

is open in $\mathcal{C}^{s}\left(\mathbf{R}^{n}\right)$. Since $\mathcal{C}^{s}\left(\mathbf{R}^{n}\right)$ is the union of such sets, we are reduced to proving the regularity of $T_{f}$ on $\mathcal{A}$. Now we note that $f \circ g=f \theta \circ g$ for $g \in \mathcal{A}$. Furthermore, by exploiting (2.4) and (2.7), it can be easily checked that $f \theta$ satisfies the assumptions of Theorem 7 whenever $f$ does. Then we can assume that $f$ has compact support, and Proposition 5 implies that $f \in \operatorname{cl}_{W^{r}(E)}\left(\mathcal{H}_{\infty}\right)$, where $E$ is:

1. $W_{\infty}^{1}(\mathbf{R})$,

2. $\mathcal{Z} \cap W_{\infty}^{1}$

3. $\mathcal{C}^{s}(\mathbf{R})(s>1$, noninteger $)$,

4. $\mathcal{C}^{s}(\mathbf{R})(s>1$, integer $)$,

respectively. According to Theorems $3,4,6$, we have

$$
\|f \circ g\|_{\mathcal{C}^{s}\left(\mathbf{R}^{n}\right)} \leq\|f\|_{E} \psi_{s}\left(\|g\|_{\mathcal{C}^{s}\left(\mathbf{R}^{n}\right)}\right),
$$

for some continuous increasing function $\psi_{s}$ from $\mathbf{R}^{+}$to itself. From (4.2), we readily deduce that

$$
\|f \circ g\|_{\mathcal{C}^{s}\left(\mathbf{R}^{n}\right)} \leq\left\|\mathcal{R}_{I} f\right\|_{E(I)} \psi_{s}\left(\|g\|_{\mathcal{C}^{s}\left(\mathbf{R}^{n}\right)}\right)
$$

for any $f \in E$ and $g \in \mathcal{A}$. By taking $f=\theta p$, where $p$ is a polynomial, we see that the hypotheses of Theorem 8 are fulfilled, with $\mathcal{X}:=\mathcal{C}^{s}\left(\mathbf{R}^{n}\right)$. According to Theorem 8, we obtain that $(f, g) \mapsto f \circ g$ is a $C^{r}$ mapping from $P_{r, E}(I) \times \mathcal{A}$ to $\mathcal{C}^{s}\left(\mathbf{R}^{n}\right)$. Since we have

$$
\mathcal{R}_{I}\left(\operatorname{cl}_{W^{r}(E)}\left(\mathcal{H}_{\infty}\right)\right) \subseteq P_{r, E}(I),
$$

Proposition 9 yields the conclusion. 


\subsection{Proof of the regularity Theorem [7, Necessity.}

4.3.1. Differentiability of $f$. We first prove that $f$ is of class $C^{1}$, by exploiting an idea of Drábek (cf. [6] p. 52]), who considered the case $0<s<1$.

Proposition 10. Let $s>0, f: \mathbf{R} \rightarrow \mathbf{R}$. If $T_{f}$ is continuous from $\mathcal{C}^{s}\left(\mathbf{R}^{n}\right)$ to itself, then $f$ is continuously differentiable.

Proof. For $0<s<1$, the result is due to Drábek [6] and, for $s>1$, it is immediate. We now assume that $s=1$. It clearly suffices to consider the case $n=1$. Let $E$ be the Banach space of functions $g: \mathbf{R} \rightarrow \mathbf{R}$ for which

$$
\|g\|_{E}:=\|g\|_{\infty}+\sup _{x \in \mathbf{R}, 0<|h| \leq 1 / e} \frac{|g(x+h)-g(x)|}{|h||\log | h||}<+\infty .
$$

By Proposition 1, our assumption implies that $T_{f}$ is continuous from $\mathcal{C}^{1}(\mathbf{R})$ to $E$. Let $\xi_{0}, x_{0}, h \in \mathbf{R}$. By Lemma 4 , the function $u_{h}$ of $\mathbf{R}$ to itself defined by

$$
u_{h}(x):=\sigma\left(x-x_{0}\right)+\xi_{0}+h, \quad \forall x \in \mathbf{R},
$$

belongs to $\mathcal{C}^{1}(\mathbf{R})$. Clearly,

$$
\lim _{h \rightarrow 0} u_{h}=u_{0}, \quad \text { in } \mathcal{C}^{1}(\mathbf{R}) .
$$

Now let $\epsilon>0$ be given arbitrarily. By continuity of $T_{f}$ at $u_{0}$, there exists $\delta>0$ depending only on $x_{0}, \xi_{0}$ such that

$$
\sup _{0<\left|x-x^{\prime}\right|<e^{-1}}\left|\frac{\left(f\left(u_{h}(x)\right)-f\left(u_{h}\left(x^{\prime}\right)\right)\right)-\left(f\left(u_{0}(x)\right)-f\left(u_{0}\left(x^{\prime}\right)\right)\right)}{\sigma\left(x-x^{\prime}\right)}\right|<\epsilon
$$

provided that $0<|h|<\delta$. Then by setting $x^{\prime}=x_{0}$, we obtain the following inequality:

$$
\left|\frac{f\left(\xi_{0}+\sigma\left(x-x_{0}\right)+h\right)-f\left(\xi_{0}+h\right)}{\sigma\left(x-x_{0}\right)}-\frac{f\left(\xi_{0}+\sigma\left(x-x_{0}\right)\right)-f\left(\xi_{0}\right)}{\sigma\left(x-x_{0}\right)}\right|<\epsilon,
$$

for $0<\left|x-x_{0}\right|<e^{-1}$, which holds whenever $0<|h|<\delta$.

By [4, p. 414], the function $f$ is locally Lipschitz continuous, and thus $f^{\prime}$ exists almost everywhere. We now show by contradiction that $f^{\prime}$ exists everywhere. Thus we assume that there exists $\xi_{0} \in \mathbf{R}$ such that

$$
\bar{L}:=\limsup _{t \rightarrow 0} \frac{f\left(\xi_{0}+t\right)-f\left(\xi_{0}\right)}{t}>\underline{L}:=\liminf _{t \rightarrow 0} \frac{f\left(\xi_{0}+t\right)-f\left(\xi_{0}\right)}{t} .
$$

Since $f$ is locally Lipschitz continuous, $\bar{L}$ and $\underline{L}$ are both finite. Let $\left\{\tau_{j}\right\}_{j \in \mathbf{N}}$, $\left\{t_{j}\right\}_{j \in \mathbf{N}}$ be sequences in $\left[-e^{-1}, e^{-1}\right] \backslash\{0\}$ converging to 0 and such that

$$
\bar{L}=\lim _{j \rightarrow \infty} \frac{f\left(\xi_{0}+t_{j}\right)-f\left(\xi_{0}\right)}{t_{j}}, \quad \underline{L}=\lim _{j \rightarrow \infty} \frac{f\left(\xi_{0}+\tau_{j}\right)-f\left(\xi_{0}\right)}{\tau_{j}} .
$$

Now let $x_{j}^{\prime}, x_{j}^{\prime \prime} \in \mathbf{R}$ be such that $\left|x_{j}^{\prime}-x_{0}\right|<e^{-1},\left|x_{j}^{\prime \prime}-x_{0}\right|<e^{-1}$, and

$$
\sigma\left(x_{j}^{\prime}-x_{0}\right)=t_{j}, \quad \sigma\left(x_{j}^{\prime \prime}-x_{0}\right)=\tau_{j} .
$$

Moreover, let $\left\{h_{l}\right\}_{l \in \mathbf{N}}$ be a sequence in $\mathbf{R} \backslash\{0\}$ such that $\lim _{l \rightarrow \infty} h_{l}=0$, and such that $f^{\prime}$ is differentiable at all points of the set $\left\{\xi_{0}+h_{l}: l \in \mathbf{N}\right\}$. Now let 
$\bar{\epsilon} \in] 0,(\bar{L}-\underline{L}) / 4[$. As we have observed above, there exists $\bar{\delta}>0$ such that (4.3) holds with $\epsilon=\bar{\epsilon}$, and for all $0<|h|<\bar{\delta}$. Then we obtain

$$
\begin{aligned}
& \left|\frac{f\left(\xi_{0}+t_{j}+h_{l}\right)-f\left(\xi_{0}+h_{l}\right)}{t_{j}}-\frac{f\left(\xi_{0}+t_{j}\right)-f\left(\xi_{0}\right)}{t_{j}}\right|<\bar{\epsilon}, \\
& \left|\frac{f\left(\xi_{0}+\tau_{j}+h_{l}\right)-f\left(\xi_{0}+h_{l}\right)}{\tau_{j}}-\frac{f\left(\xi_{0}+\tau_{j}\right)-f\left(\xi_{0}\right)}{\tau_{j}}\right|<\bar{\epsilon},
\end{aligned}
$$

for all $j \in \mathbf{N}$, and for all $l \in \mathbf{N}$ such that $\left|h_{l}\right|<\bar{\delta}$. Now we let $j$ tend to infinity in (4.4), and we obtain

$$
\left|f^{\prime}\left(\xi_{0}+h_{l}\right)-\bar{L}\right| \leq \bar{\epsilon}, \quad\left|f^{\prime}\left(\xi_{0}+h_{l}\right)-\underline{L}\right| \leq \bar{\epsilon}
$$

for all $l \in \mathbf{N}$ such that $\left|h_{l}\right|<\bar{\delta}$. Accordingly, $|\bar{L}-\underline{L}| \leq 2 \bar{\epsilon}$, a contradiction. Thus we conclude that $f^{\prime}\left(\xi_{0}\right)$ exists for all $\xi_{0} \in \mathbf{R}$. We now prove that $f^{\prime}$ is continuous at $\xi_{0}$. Let $\epsilon>0$ be given arbitrarily. Then there exists $\delta>0$ such that (4.3) holds, and by taking the limit as $x$ tends to $x_{0}$ in (4.3), we obtain

$$
\left|f^{\prime}\left(\xi_{0}+h\right)-f^{\prime}\left(\xi_{0}\right)\right| \leq \epsilon
$$

for $0<|h|<\delta$. Thus $f^{\prime}$ is continuous at $\xi_{0}$.

4.3.2. The case $r=0, s=1$. The following result relies upon ideas of Sobolevskij [16], who considered Hölder spaces.

Proposition 11. Let $f: \mathbf{R} \rightarrow \mathbf{R}$. If $T_{f}$ is continuous from $\mathcal{C}^{1}\left(\mathbf{R}^{n}\right)$ to itself, then we have

$$
\lim _{t \rightarrow 0+}\left(\sup _{\xi \in K} \frac{|f(\xi+t)+f(\xi-t)-2 f(\xi)|}{\gamma(t)}\right)=0,
$$

for all compact subsets $K$ of $\mathbf{R}$.

Proof. By Proposition 10 the function $f$ is continuously differentiable. Then, for all $\eta>0$, the averaged function $f_{\eta}$, defined by

$$
f_{\eta}(\xi):=\frac{1}{\eta} \int_{\xi}^{\xi+\eta} f(\tau) d \tau=\int_{0}^{1} f(\xi+\tau \eta) d \tau \quad \forall \xi \in \mathbf{R},
$$

is of class $C^{2}$. Formula (2.7) implies that

$$
\lim _{t \rightarrow 0+}\left(\sup _{\xi \in K} \frac{\left|f_{\eta}(\xi+t)+f_{\eta}(\xi-t)-2 f_{\eta}(\xi)\right|}{\gamma(t)}\right)=0,
$$

for all compact intervals $K$ of $\mathbf{R}$. Now we set

$$
g_{\xi}(x):=\xi+\sigma(x) .
$$

Since $\sigma$ is odd, we have $g_{\xi}( \pm h)=\xi \pm \sigma(h)$, for all $h, \xi \in \mathbf{R}$. Now we assume that $0<t<1 / e$. Clearly, there exists a unique number $h \in] 0,1 / e[$ such that $t=h|\log h|$. By (3.7), we have $\gamma(t) \geq h$. From the identities

$$
\left(f-f_{\eta}\right)(\xi+\epsilon t)=\int_{0}^{1}\left(f\left(g_{\xi}(\epsilon h)\right)-f\left(g_{\xi+\tau \eta}(\epsilon h)\right)\right) d \tau \quad(\epsilon=-1,0,1),
$$


we deduce that

$$
\begin{aligned}
& \sup _{\xi \in K} \mid\left(f-f_{\eta}\right)(\xi+t)+\left(f-f_{\eta}\right)(\xi-t)- 2\left(f-f_{\eta}\right)(\xi) \mid \\
& \leq \gamma(t) \sup _{\xi \in K} \sup _{0 \leq \tau \leq 1}\left\|f \circ g_{\xi+\tau \eta}-f \circ g_{\xi}\right\| .
\end{aligned}
$$

Then by (4.5), we obtain

$$
\limsup _{t \rightarrow 0+}\left(\sup _{\xi \in K} \frac{|f(\xi+t)+f(\xi-t)-2 f(\xi)|}{\gamma(t)}\right) \leq \sup _{\xi \in K} \sup _{0 \leq \tau \leq 1}\left\|f \circ g_{\xi+\tau \eta}-f \circ g_{\xi}\right\|,
$$

for each fixed $\eta>0$. By exploiting the uniform continuity of $T_{f}$ on the compact subset

$$
\left\{g_{\xi+y}: \xi \in K, y \in[0,1]\right\}
$$

of the space $\mathcal{C}^{1}(\mathbf{R})$, we see that the above right-hand side is arbitrarily small with $\eta$.

4.3.3. The case $r=0, s>1$. By arguing as in the proof of [11 Prop. 4.21], we have the following.

Proposition 12. Let $s>1$. Let $f: \mathbf{R} \rightarrow \mathbf{R}$. If the operator $T_{f}$ is continuous from $\mathcal{C}^{s}\left(\mathbf{R}^{n}\right)$ to itself, then $f \theta$ belongs to the closure of $C_{b}^{\infty}$ in $\mathcal{C}^{s}$, for all $\theta \in \mathcal{D}(\mathbf{R})$.

Proof. It clearly suffices to consider the case $n=1$. According to section $4.2, T_{\theta}$ is continuous on $\mathcal{C}^{s}(\mathbf{R})$, for all $\theta \in \mathcal{D}(\mathbf{R})$. Since $\mathcal{C}^{s}(\mathbf{R})$ is a Banach algebra, $T_{f \theta}$ is also continuous on $\mathcal{C}^{s}(\mathbf{R})$. Thus we can assume that $f$ has compact support and show that $f$ itself belongs to the closure of $C_{b}^{\infty}$ in $\mathcal{C}^{s}$. Let $R>0$ be such that $f(x)=0$ for $|x| \geq R$. Let $\psi \in C_{b}^{\infty}$ be such that $\psi(x)=x$ for $|x| \leq R$ and $|\psi(x)|>R$ for $|x|>R$. Since $f$ is bounded, we already know that $\left(f * \varphi_{j}\right) \circ \psi \in C_{b}^{\infty}$ (cf. section 2.4). We now note that the maps $y \mapsto \psi-y$ and $\varphi_{j}$ are continuous from $\mathbf{R}$ to $\mathcal{C}^{s}(\mathbf{R})$, and from $\mathbf{R}$ to itself, respectively. By our assumption, the map $F_{j}$ of $\mathbf{R}$ to $\mathcal{C}^{s}(\mathbf{R})$ defined by

$$
F_{j}(y):=(f \circ(\psi-y)-f \circ \psi) \varphi_{j}(y)
$$

is continuous and satisfies $\lim _{y \rightarrow \infty}\left\|F_{j}(y)\right\| y^{k}=0$ for all $k \in \mathbf{N}$. Then $F_{j}$ is Bochner integrable, and we have

$$
\left\|\int_{\mathbf{R}} F_{j}(y) d y\right\| \leq \int_{\mathbf{R}}\left\|F_{j}(y)\right\| d y .
$$

For all $\delta>0$, one has

$$
\int_{\mathbf{R}}\left\|F_{j}(y)\right\| d y \leq\|\varphi\|_{1} \sup _{|y| \leq \delta}\|f \circ(\psi-y)-f \circ \psi\|+2\|f\|_{\infty} \int_{|y|>\delta}\left|\varphi_{j}(y)\right| d y .
$$

By exploiting the fact that $\lim _{j \rightarrow \infty} \int_{|y|>\delta}\left|\varphi_{j}(y)\right| d y=0$ and the continuity of $T_{f}$ on $\mathcal{C}^{s}\left(\mathbf{R}^{n}\right)$, we obtain

$$
\lim _{j \rightarrow \infty} \int_{\mathbf{R}}\left\|F_{j}(y)\right\| d y=0 .
$$

Going back to (4.6), we see that

$$
\lim _{j \rightarrow \infty}\left(f * \varphi_{j}\right) \circ \psi=f \circ \psi \quad \text { in } \mathcal{C}^{s}(\mathbf{R}) .
$$


Since $f \circ \psi=f$, we conclude that $f$ belongs to the closure of $C_{b}^{\infty}(\mathbf{R})$ in $\mathcal{C}^{s}(\mathbf{R})$.

4.3.4. End of the proof of Theorem \%. Assume that $T_{f}$ is $r$ times continuously differentiable from $\mathcal{C}^{s}\left(\mathbf{R}^{n}\right)$ to itself. We first consider the case $s=1$ and prove, by induction on $r$, that $f$ satisfies condition 2 of Theorem 7. By Propositions 10 and 11 we know that the statement holds for $r=0$. Thus we now assume that the statement holds for $r$, and we prove it for $r+1$. We argue as in [11 p. 474]. Let $g \in \mathcal{C}^{1}\left(\mathbf{R}^{n}\right), h \in \mathcal{C}^{1}\left(\mathbf{R}^{n}\right) \backslash\{0\}$. The differentiability of $T_{f}$ at $g$ implies that

$$
\lim _{t \rightarrow 0}\left\|\frac{f \circ(g+t h)-f \circ g}{t\|h\|}-d T_{f}[g]\left(\frac{h}{\|h\|}\right)\right\|=0 .
$$

Since we already know that $f^{\prime}$ exists at all points of $\mathbf{R}$, we deduce that

$$
\left(f^{\prime} \circ g\right) h=d T_{f}[g](h), \quad \forall h \in \mathcal{C}^{1}\left(\mathbf{R}^{n}\right) .
$$

Since $T_{f}$ is $(r+1)$ times continuously differentiable, then $d T_{f}[\cdot]$ is $r$ times continuously differentiable from $\mathcal{C}^{1}\left(\mathbf{R}^{n}\right)$ to $\mathcal{L}\left(\mathcal{C}^{1}\left(\mathbf{R}^{n}\right), \mathcal{C}^{1}\left(\mathbf{R}^{n}\right)\right)$, and since the evaluation map $A \mapsto A[1]$ at the constant function $1 \in \mathcal{C}^{1}\left(\mathbf{R}^{n}\right)$ is linear and continuous from $\mathcal{L}\left(\mathcal{C}^{1}\left(\mathbf{R}^{n}\right), \mathcal{C}^{1}\left(\mathbf{R}^{n}\right)\right)$ to $\mathcal{C}^{1}\left(\mathbf{R}^{n}\right)$, we conclude that the map $g \mapsto T_{f}[g](1)=f^{\prime} \circ g$ is $r$ times continuously differentiable from $\mathcal{C}^{1}\left(\mathbf{R}^{n}\right)$ to itself. Then, by the inductive assumption, $f^{\prime}$ satisfies condition 2 of Theorem 7 , and thus $f$ satisfies the same condition with $r+1$ instead of $r$. The case $s>1$ can be treated similarly by exploiting Propositions 5 and 12. We now consider the case $0<s<1$. The same inductive argument as in the case $s=1$ reduces the proof to the case $r=0$. But the case $r=0$ can be treated by a straightforward modification of the proof of Drábek [6].

\section{REFERENCES}

[1] J. Appell and P.P. Zabreiko, Nonlinear Superposition Operators, Cambridge Tracts in Mathematics 95, Cambridge University Press, Cambridge (1990). MR 91k:47168

[2] C. Bennett and R. Sharpley, Interpolation of Operators, Pure and Applied Mathematics 129, Academic Press, Boston (1988). MR 89e:46001

[3] G. Bourdaud, Le calcul fonctionnel dans les espaces de Sobolev, Invent. Math., 104 (1991), pp. 435-446. MR 93b:46053

[4] G. Bourdaud, Fonctions qui opèrent sur les espaces de Besov et de Triebel, Ann. Inst. Henri Poincaré, Analyse non linéaire, 10 (1993), pp. 413-422. MR 94m:46055

[5] G. Bourdaud, Analyse fonctionnelle dans l'espace Euclidien, 2ième édition, Pub. Math. Univ. Paris 7 (1995). MR 89a:46001 (1st ed.)

[6] P. Drábek, Continuity of Nemyckij's operator in Hölder spaces, Comm. Math. Univ. Carolinae, 16 (1975), pp. 37-57. MR 52:1447

[7] G.B. Folland, Real analysis, modern techniques and their applications, John Wiley and Sons, New York (1984). MR 86k:28001

[8] Y. Katznelson, An introduction to Harmonic Analysis, Dover, New York (1976). MR 54:10976

[9] H. Koch and W. Sickel, Pointwise multipliers of Besov spaces of smoothness zero and spaces of continuous functions, Rev. Math. Iberoamericana (to appear).

[10] A. Kufner, O. John and S. Fučik, Function Spaces, Noordhoff International Publishing, Leyden (1977). MR 58:2189

[11] M. Lanza de Cristoforis, Higher order differentiability properties of the composition and of the inversion operator, Indag. Matem. N.S., 5 (1994), pp. 457-482. MR 95j:47081

[12] M. Lanza de Cristoforis, Differentiability properties of an abstract autonomous composition operator, J. London Math. Soc. 61 (2000), pp. 923-936. MR 2001c:47069

[13] A. Marchaud, Sur les dérivées et sur les différences des fonctions des variables réelles, J. Math. Pure et Appl. 6 (1927), pp. 337-425. 
[14] J. Peetre, New Thoughts on Besov spaces, Duke University Mathematics Series, No. 1. Mathematics Department, Duke University, Durham, N.C. (1976). MR 57:1108

[15] T. Runst and W. Sickel, Sobolev Spaces of Fractional Order, Nemytskij Operators, and Nonlinear Partial Differential Equations, De Gruyter, Berlin (1996). MR 98a:47071

[16] E. P. Sobolevskij, The superposition operator in Hölder spaces, Voronezh, VINITI No. 376584 (1984), (in Russian).

[17] P.M. Tamrazov, Structural and approximational properties of functions in the complex domain, Linear Spaces and Approximation, Birkhäuser, Basel, (1978), pp. 503-514. MR 80a:30044

[18] P.M. Tamrazov, Divided differences and moduli of smoothness of functions, function superpositions and their application, Constructive theory of functions 1984, Sofia, 1984, pp. 840-851.

[19] H. Triebel, Theory of Function Spaces II, Birkhäuser, Basel (1992). MR 93f:46029

[20] S.E. Warschawski, On the higher derivatives at the boundary in conformal mapping, Trans. Amer. Math. Soc. 38 (1935), pp. 310-340.

[21] A. Zygmund, Trigonometric Series, Cambridge University Press, New York (1959). MR 21:6498

Institut de Mathématiques de Jussieu, Équipe D’Analyse Fonctionnelle, Case 186, 4 Place Jussieu, 75252 Paris Cedex 05, France

E-mail address: bourdaud@ccr.jussieu.fr

University of Padova, Dipartimento di Matematica Pura ed Applicata, Via Belzoni 7, 35131 Padova, Italia

E-mail address: mldc@math.unipd.it 